\title{
Tricuspid Valve Vena Contracta Area
}

National Cancer Institute

\section{Source}

National Cancer Institute. Tricuspid Valve Vena Contracta Area. NCI Thesaurus. Code C127601.

The area of the vena contracta of the tricuspid valve. 